\title{
Profiles of Sugar and Organic Acid of Fruit Juices: A Comparative Study and Implication for Authentication
}

\author{
Jiaxiu Li, Chunling Zhang, Hui Liu, Jiechao Liu, and Zhonggao Jiao (iD \\ Zhengzhou Fruit Research Institute, Chinese Academy of Agricultural Sciences, Zhengzhou 450009, China \\ Correspondence should be addressed to Zhonggao Jiao; jiaozhonggao@caas.cn
}

Received 13 March 2020; Revised 1 August 2020; Accepted 6 August 2020; Published 28 August 2020

Academic Editor: Elena Gonz lez Fandos

Copyright (c) 2020 Jiaxiu Li et al. This is an open access article distributed under the Creative Commons Attribution License, which permits unrestricted use, distribution, and reproduction in any medium, provided the original work is properly cited.

\begin{abstract}
A comparison of sugar and organic acid profiles among different fruit juices (including apple, pear, peach, grape, sweet cherry, strawberry, and blueberry with various varieties) was performed to assess the possibility for authentication coupled with chemometrics. It was found that the distribution of each sugar and organic acid in juices showed some specific characteristics related to fruit species, despite the fact that great differences in the content existed among different varieties. Sucrose was the most abundant sugar in peach juice, accounting for 58.26-77.11\% of the total sugar content. However, in grape, blueberry, and sweet cherry juice, glucose and fructose were the predominant sugars. Pear juice contained the highest level of sorbitol, which contributed to $15.02-43.07 \%$ of the total sugar content. Tartaric acid was detected only in grape juice among the seven species of fruit juice, with a proportion of $57.95-89.68 \%$ in the total acid content. Malic acid was the predominant organic acid in apple and sweet cherry juice, accounting for $69.92-88.30 \%$ and $97.51-98.73 \%$ of the total acid content of each species. Citric acid was the predominant organic acid in strawberry and blueberry juice, which contributed to $62.39-83.73 \%$ and $73.36-89.56 \%$ of the total acid content of each species. With the aid of principal component analysis and linear discriminant analysis (LDA), the juice samples could be successfully classified according to fruit species by using the sugar and/or organic acid composition as analytical data. Combination of sugar and organic acid composition gave the best differentiation of these seven species of juices, with a $100 \%$ correct classification rate for both the original and the cross-validation method in LDA. Adding malic/citric into the dataset of the organic acid content may also improve the differentiation effect. Furthermore, the adulteration of sweet cherry juice, blueberry juice, raspberry juice, and grape juice with apple juice, pear juice, or peach juice could also be distinguished from their corresponding pure juices based on sugar and organic acid composition by LDA.
\end{abstract}

\section{Introduction}

Sugars and organic acids are the main nutrients and taste components in fruit juices, which contribute to the main soluble solid content and sensory properties of fruit juices $[1,2]$. During processing and storage of fruit juices, the sugars and organic acids show a lower susceptibility to changes as compared with other components such as pigments, antioxidants, and flavor compounds $[3,4]$. Therefore, characterization of the composition of sugar and/or organic acid in fruit juices may be a desirable approach for fruit juice authenticity as well as quality control. Based on sugar or organic acid profiles, several commercial fruit juices (including apple, grape, mandarin, orange, and pineapple juice) were distinguished according to the fruit species by linear discriminant analysis [5-7]. The differentiation of fresh Greek orange juice made from the Merlin cultivar according to the geographical origin was also achieved by using linear discriminant analysis based on composition of sugar and organic acid [8]. However, many other fruit juice matrices (such as pear, peach, strawberry, blueberry, and sweet cherry) were not included. In order to get comprehensive understanding on the differences in sugar and organic acid profiles among various fruit juices and their implication for authentication, more comparative studies on sugar and organic acid profiles of various fruit juices are still needed.

High-performance liquid chromatography (HPLC) is a popular method for the analysis of food composition due to its high precision and analytical selectivity. It can provide rapid quantitative separation of many components in 
various food matrices, such as carbohydrates [9], organic acids [10], phenolics [11], and vitamins [12]. Several researches have revealed the compositions of sugars or organic acids in fruit juices by using the HPLC technique owing to its ability to determine and quantify the main sugars and organic acids in fruit juices $[8,13-16]$.

Besides fruit species, variety is also a crucial factor that affects the composition and content of sugar and organic acid in fruit juices [17-20]. Accordingly, it is also necessary to compare the sugar and organic acid profiles of fruit juices prepared from different varieties, with the aim of finding the common pattern among different varieties and their implication for authentication. However, the information about the variety of most commercial fruit juice products is not available, and the authentication of the collected commercial samples is not so easy. In the present study, the laboratory-made fruit juices of apple, pear, peach, grape, sweet cherry, strawberry, and blueberry with various varieties were used to determine the composition of sugar and organic acid by high-performance liquid chromatography, and a comparison among different fruit juices was performed to find their implication for authentication with the aid of chemometrics.

\section{Materials and Methods}

2.1. Fruit Materials. Fruits of apple (12 varieties), pear (15 varieties), peach (12 varieties), grape (17 varieties), sweet cherry (14 varieties), and strawberry (10 varieties) were harvested at maturity from the orchard of the Zhengzhou Fruit Research Institute, Chinese Academy of Agricultural Sciences, which is located in the Henan Province of the Central China. The blueberry (5 varieties) was obtained from Linyi of Shandong Province, China. Each variety of fruit juice was recorded as a sample.

2.2. Chemicals and Reagents. Standards of sucrose, glucose, fructose, sorbitol, oxalic acid, tartaric acid, quinic acid, malic acid, shikimic acid, citric acid, and fumaric acid, all with a purity exceeding $99.0 \%$, and lactic acid with a purity exceeding 98.0\% were purchased from Sigma-Aldrich (St. Louis, MO, USA). Ethylenediaminetetraacetic acid calcium disodium salt hydrate (Ca-EDTA) was purchased from Aladdin Industrial Corporation (Shanghai, China). $\mathrm{H}_{3} \mathrm{PO}_{4}$ and $\left(\mathrm{NH}_{4}\right)_{2} \mathrm{HPO}_{4}$ of the analytical grade were purchased from Zhiyuan Chemical Reagent Co., LTD (Tianjin, China) and Kemiou Chemical Reagent Co., LTD (Tianjin, China), respectively.

2.3. Preparation of Fruit Juice. The fruits of apple, pear, peach, grape, sweet cherry, strawberry, and blueberry were washed, cut into pieces, pitted if necessary, and then pureed using a lab-scale food processor (JYL-C52V, Joyoung, China). After pasteurization in boiling water for $5 \mathrm{~min}$, the puree was cooled and treated with pectinase $(0.1 \%,>40 \mathrm{PA} /$ $\mathrm{mg}$, DSM, Germany) at $50^{\circ} \mathrm{C}$ for $40 \mathrm{~min}$ and then centrifuged at $4000 \mathrm{rpm}$ for $10 \mathrm{~min}$ with a centrifuge (L-550, Xiangyi Centrifuge Instrument Co., Ltd., Hunan, China). The resulted supernatant was recovered and stored at $-80^{\circ} \mathrm{C}$ for HPLC analysis.

To assess the possibility of detecting adulteration of higher-cost fruit juices with cheaper alternatives, the randomly selected sweet cherry juice, blueberry juice, grape juice, and strawberry juice were mixed with $20 \%, 40 \%, 60 \%$, and $80 \%$ of apple juice, pear juice, or peach juice, respectively.

2.4. HPLC Analysis of Sugar and Organic Acid. HPLC analysis of the soluble sugars in fruit juices was performed by using a 1525 binary HPLC system coupled with a RI 2414 refractive index detector (Waters Corp., Wilford, MA, USA). The soluble sugars were separated by a Waters Sugar-Pak I column $(6.5 \times 300 \mathrm{~mm})$ at $80^{\circ} \mathrm{C}$ with Ca-EDTA solution $(50 \mathrm{mg} /$ $\mathrm{L})$ as the elution solvent. The injection volume was $10 \mu \mathrm{L}$, and the flow rate of the elution solvent was $0.5 \mathrm{~mL} / \mathrm{min}$. Sucrose, glucose, fructose, and sorbitol were used as standards to identify and quantify the soluble sugars in fruit juices. All the standards could be detected in a total run time of $20 \mathrm{~min}$ (Figure S1). Ten concentrations in a range of $0.042-41.64 \mathrm{mg} /$ $\mathrm{mL}$ for sucrose, $0.038-38.06 \mathrm{mg} / \mathrm{mL}$ for glucose, $0.040-40.20 \mathrm{mg} / \mathrm{mL}$ for fructose, and $0.024-24.06 \mathrm{mg} / \mathrm{mL}$ for sorbitol were used to create a standard curve for calculating the content of each sugar in fruit juices.

As for organic acid analysis, a 1525 binary HPLC system coupled with a 2998 photodiode array detector (Waters Corp., Wilford, MA, USA) and Ultimate ${ }^{\circledR}$ AQ-C18 $(4.6 \times 250 \mathrm{~mm}, 5 \mu \mathrm{m}$, Welch Science \& Technology Co., Ltd, Shanghai, China) were used with the diammonium hydrogen phosphate solution $(0.02 \mathrm{~mol} / \mathrm{L}$, adjusted to $\mathrm{pH} 2.4$ with phosphoric acid) as the elution solvent. The injection volume was $10 \mu \mathrm{L}$, and the flow rate of the elution solvent was $1.0 \mathrm{~mL} / \mathrm{min}$. The chromatograms were recorded at $210 \mathrm{~nm}$. Oxalic acid, tartaric acid, quinic acid, malic acid, shikimic acid, lactic acid, citric acid, and fumaric acid were used as standards to identify and quantify the organic acids in fruit juices. All the organic acid standards could be detected in a total run time of $10 \mathrm{~min}$ (Figure S1). Ten concentrations in a range of $2.5-1000 \mathrm{mg} / \mathrm{L}$ for oxalic acid, $25-1350 \mathrm{mg} / \mathrm{L}$ for tartaric acid, $102.5-6150 \mathrm{mg} / \mathrm{L}$ for quinic acid, $77.5-6150 \mathrm{mg} / \mathrm{L}$ for malic acid, $0.44-160 \mathrm{mg} / \mathrm{L}$ for shikimic acid, $41.6-2600 \mathrm{mg} / \mathrm{L}$ for lactic acid, $12-4620 \mathrm{mg} / \mathrm{L}$ for citric acid, and $0.065-260 \mathrm{mg} / \mathrm{L}$ for fumaric acid were used to create a standard curve for calculating the content of each organic acid in fruit juices.

2.5. Statistical Analysis. Statistical analysis was performed with SPSS Statistics 22.0 software (IBM Corp., Armonk, NY, USA). The mean value for the triplicate of each sample was used for the comparison and chemometric study. One-way analysis of variance (ANOVA) and Duncan's tests were used to discern the significant differences at a level of $p<0.05$.

2.6. Chemometric Analysis. The principal component analysis- (PCA-) based unsupervised method and linear discriminant analysis- (LDA-) based supervised method were 
performed with SPSS Statistics 22.0 software (IBM Corp., Armonk, NY, USA) by using different datasets: (a) sugar content; (b) sugar content combined with Fru/Glu; (c) organic acid content; (d) organic acid content combined with malic/citric; (e) sugar content combined with organic acid content.

\section{Results and Discussion}

3.1. Sugar Profiles of Different Fruit Juices. The sugar composition and content of different fruit juices are summarized in Table 1. In spite of the great differences in contents of individual sugars among different varieties (Tables S1-S7), a common pattern of sugar composition was still observed as compared with other species of fruit juice.

Sucrose was the most abundant sugar in peach juice, accounting for $58.26-77.11 \%$ of the total sugar content, which was in agreement with the results obtained from the peach fruit of different cultivars [21, 22]. However, in grape juice and blueberry juice, the sucrose content was quite lower, and no sucrose could be detected in sweet cherry juice. In the above three species of fruit juices, glucose and fructose were the predominant sugars, which were also in accordance with the results obtained from fruit samples [23-25]. The sucrose content in apple, pear, and strawberry juice was in the range of $8.99-38.39,4.07-68.92$, and $4.56-31.12 \mathrm{~g} / \mathrm{L}$, respectively, which contributed to $8.70-28.87 \%, 3.40-54.82 \%$, and $9.53-40.43 \%$ of the total sugar content in each fruit juice (Tables S1, S2, and S6), indicating a wider range of content distribution in these three species of fruit juice. Therefore, based on the sucrose content and its proportion in the total sugar content, the seven species of fruit juice could be classified into three categories: one is peach juice, which represents a high content of sucrose in total sugar; another class includes apple, pear, and strawberry juice, with a medium content of sucrose in total sugar; the other three fruit juices (grape, sweet cherry, and blueberry) were characterized as few sucrose content in soluble sugars.

Glucose and fructose existed in all the fruit juices with a relatively high content, but the ratio of fructose to glucose (Fru/Glu) as well as their proportions in the total sugar content varied according to the species of fruit juice. As shown in Tables S1 and S2, the Fru/Glu ratio of apple and pear juice was in the range of $1.59-3.43$ and $1.46-4.08$, respectively, rather higher than those of the other five species of fruit juice, which could enable the differentiation of these two species from other juices. The lowest Fru/Glu ratio was observed in sweet cherry juice with a range of $0.73-0.93$ (Table S5). However, in peach, strawberry, and blueberry juice, the Fru/Glu ratios were all above 1.0 (Tables S3, S6, and S7). The Fru/Glu ratio of grape juice was also around 1.0, but some varieties of juices showed a lower ratio than 1.0 (Table S4). Evaluating the proportion in the total sugar content shows that the fructose content contributed to $52.37-60.76 \%$ of the total sugar content in different varieties of apple juice (Table S1), while the proportion was $19.73-53.57 \%$ in different varieties of pear juice (Table S3), which provided a basis for differentiation of most varieties of these two species of fruit juice. As a matter of fact, the Fru/ Glu ratio is usually suggested as an important index for the evaluation of the product quality and authenticity in fruit juices $[5,18,26]$.

Sorbitol was detected in all the varieties of apple, pear, peach, and sweet cherry juice, but there were great differences in the content among these four species of fruit juice, showing a significant difference among pear, sweet cherry, and apple or peach juice $(p<0.05)$. As shown in Table 1 , pear juice contained the highest level of sorbitol among them, which contributed to $15.02-43.07 \%$ of the total sugar content. Sweet cherry juice showed a medium level of sorbitol, with a concentration range of $7.50-25.86 \mathrm{~g} / \mathrm{L}$ and a proportion range of 9.24-15.29\% (Table S5). As for apple and peach juice, both the sorbitol concentration and proportion in the total sugar content were rather lower than those of pear and sweet cherry juice. No sorbitol was detected in other three species of fruit juice. These results are in agreement with the previous report by Eksi and Karav [27], which indicated that the differences in the sorbitol content among different species of fruit juice could be meaningful to prove the existence of foreign fruit juices by comparing 96 natural fruit juice samples obtained from 9 different species. On the basis of the sorbitol content, the identification of adulteration of apple juice with pear juice and addition of apple juice in grape juice was also investigated by other researchers $[28,29]$.

\subsection{Organic Acid Profiles of Different Fruit Juices. Similar to} the sugar profiles, the contents of organic acids in fruit juices varied among different varieties (Tables S1-S7), but there were also some distinct characteristics related to specific species of fruit juice.

As shown in Table 2, tartaric acid was detected only in grape juice among the seven species of fruit juice, with a proportion of $(74.57 \pm 9.57) \%$ in the total acid content, which indicated it could be a useful index for differentiation of grape juice from other fruit juices. Other researchers also revealed tartaric acid as the major organic acid in grape juice $[14,30,31]$ and suggested it as an indicator of adulteration with grape juice in other juices $[6,32]$. However, Ehling and Cole [32] reported the existence of tartaric acid in pomegranate juice with a concentration range of $1-5 \mathrm{mg} / \mathrm{L}$, but it was much lower than that of grape juice (above $1000 \mathrm{mg} / \mathrm{L}$ ), which had little effect on the discrimination of pomegranate juice from adulterated products with grape juice.

Quinic acid was detected in all varieties of apple, pear, peach, and blueberry juice with a concentration range of $36.53-291.85 \mathrm{mg} / 100 \mathrm{~mL}$ (Tables S1-S3 and S7), which differentiated them from other three species of fruit juice that had no quinic acid detected. Ehling and Cole also reported the existence of quinic acid in apple juice with a rather higher concentration $(40.6 \pm 0.5 \mathrm{mg} / 100 \mathrm{~mL})$ than grape juice (not detected in white grape juice and $0.363 \pm 0.010 \mathrm{mg} / 100 \mathrm{~mL}$ in red grape juice) [32], which was similar to our results. The concentration of quinic acid in pear juice prepared from different varieties was also reported to be in the range of $90-260 \mathrm{mg} / 100 \mathrm{~mL}$ by Gao et al. [33]. 


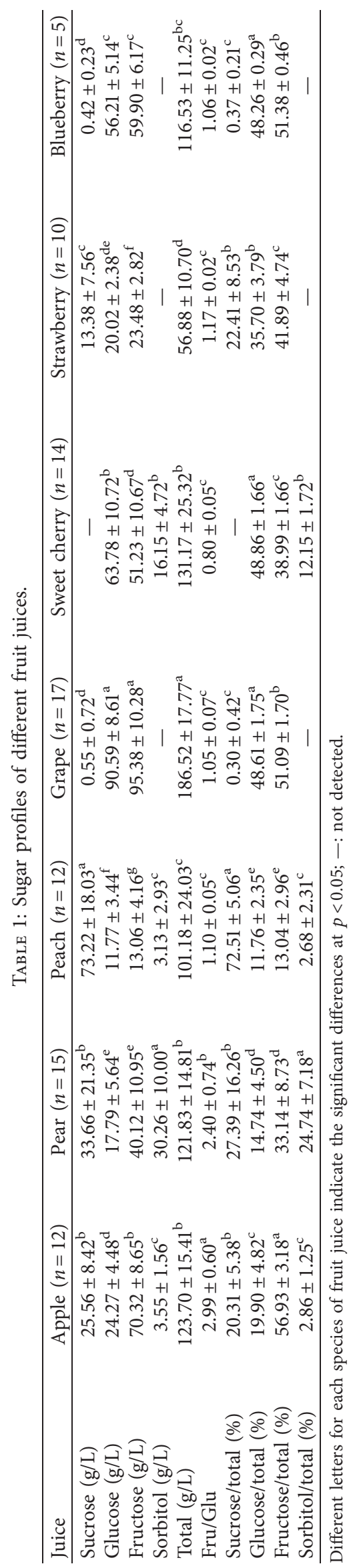




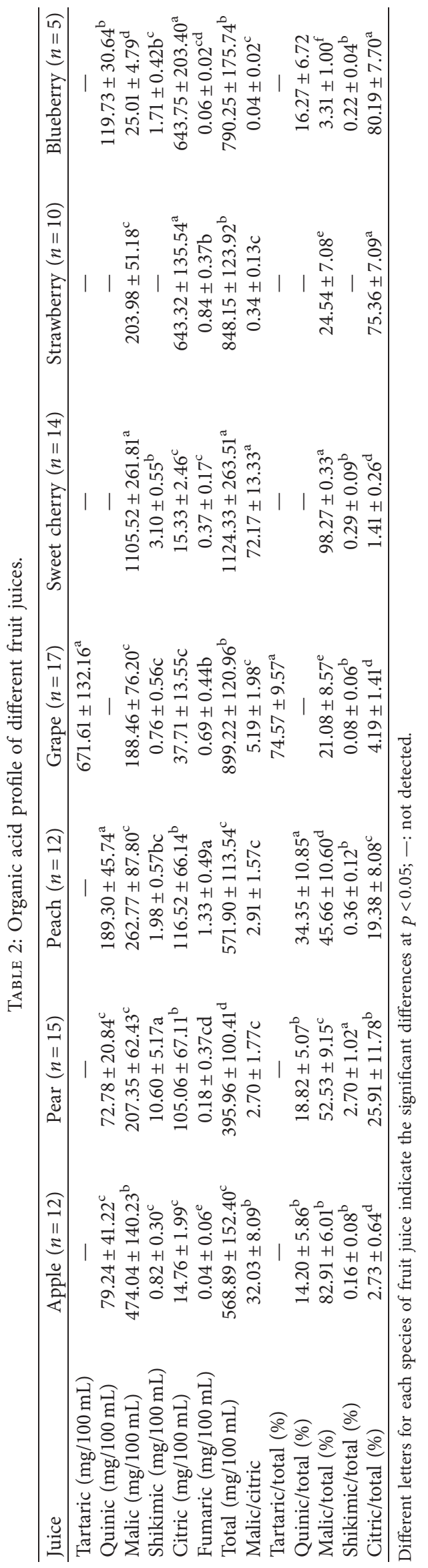


Researches on the organic acid profile in the fruits of peach and blueberry also implied the existence of quinic acid in juices of peach and blueberry [21, 34].

Malic acid and citric acid are the most abundant organic acids in fruit juices. As shown in Table 2, all the fruit juices contained a relatively high concentration of malic acid and citric acid, but there were also obvious differences among different species of fruit juice. In apple and sweet cherry juice, malic acid was the predominant organic acid, accounting for $69.92-88.30 \%$ and $97.51-98.73 \%$ of the total acid content of each species (Tables S1 and S5), which could facilitate the differentiation of these two species of juice from other species. However, in pear juice, there were two varieties that had a higher content of citric acid than that of malic acid (Table S2). In addition, the proportion of citric acid in the total acid content of pear juice was rather higher than that of apple juice, which could enable the differentiation of these two species of fruit juice. The content of malic acid in peach juice was also higher than that of citric acid except for one variety (Table S3) with a malic/citric (the ratio of malic acid to citric acid) range of 0.81-5.68, which was similar to pear juice. Moreover, three varieties of peach juice contained a higher content of quinic acid than malic or citric acid (Table S3). These results further confirmed the effect of variety on organic acid composition of fruit juices. Citric acid was the predominant organic acid in strawberry and blueberry juice, which contributed to $62.39-83.73 \%$ and $73.36-89.56 \%$ of the total acid content of each species. This unique characteristic may facilitate the differentiation of strawberry and blueberry juice from the other five species of fruit juice. Furthermore, the proportion of malic acid in the total acid content of strawberry juice was far higher than that of blueberry juice, which could be used for the discrimination of these two species of fruit juice.

Shikimic acid existed in all the fruit juices except in strawberry juice, but the concentration was rather low. Among them, pear juice had the highest content of shikimic acid, with a concentration range of $3.43-21.75 \mathrm{mg} / 100 \mathrm{~mL}$ and proportion in the total acid content range of $0.85-4.05 \%$ (Table S2), which could facilitate the differentiation of pear juice from the other five species of fruit juice. Gao et al. [26] also reported a shikimic acid concentration range of $5.7-17.3 \mathrm{mg} / 100 \mathrm{~mL}$ in pear juice prepared from different varieties, which was similar to our results. Investigations on the organic acid composition in the fruits of apple, peach, and sweet cherry also implied the existence of shikimic acid in juices of apple, peach, and sweet cherry [35-37].

Fumaric acid was detected in all the seven species of fruit juice with trace content $(0.002-2.18 \mathrm{mg} / 100 \mathrm{~mL})$. According to previous reports, fumaric acid in fruit juice was formed mainly as the result of heat treatment and microbial contamination [38], so the fumaric acid content or its proportion in the total acid content may not be considered as a characteristic index for juice authentication. However, the fumaric acid level in fruit juice was preferred as an indicator of microbial spoilage of fruits used as a raw material [38].
3.3. Unsupervised Classification of Fruit Juices by Principal Component Analysis. Principal component analysis (PCA) is the most commonly used unsupervised methods in food authentication analysis, which can reduce the dimensions of the data and make them visualized [39]. In the present research, the data of sugar and/or organic acid composition obtained from all the 85 juice samples were subjected to PCA, and the score plots of the first two principal components for different datasets are shown in Figure 1.

When the sugar content was used as analytical data for PCA, the first two principal components accounted for $85.739 \%$ of the total variance, and the juice samples were clustered into seven groups according to fruit species except for one sweet cherry juice sample which positioned close to the apple juice group in the score plots (Figure 1(a)). These results suggest that sugar composition coupled with PCA could be used to differentiate juices of apple, pear, peach, grape, strawberry, blueberry, and sweet cherry for quality control or authentication. Since the Fru/Glu ratios among different species of fruit juice showed distinct difference (Table 2), the data of Fru/Glu were then added into the dataset of the sugar content for further PCA with the attempt to improve the classification effect. As shown in Figure 1(b), one apple juice sample was misclassified in the pear juice group, and several pear juice samples positioned close to the apple juice group in the score plots, indicating an inferior classification effect when the sugar content combined with Fru/Glu was used as analytical data for PCA.

As the analytical data of the organic acid content was used for PCA, the first two principal components accounted for $59.767 \%$ of the total variance, and the juice samples were clustered into seven groups according to fruit species, even though several apple, pear, and peach juice samples were misclassified or partly overlapped in the score plots (Figure 1(c)). By comparing Figure 1(c) with Figure 1(a), a better separation of seven groups was obtained with sugar composition than with organic acid composition. Interestingly, the separation of different species of fruit juice was improved by adding the data of malic/citric into the dataset of the organic acid content for PCA. As shown in Figure 1(d), only some peach juice samples were misclassified in the pear juice group in the score plots when the data of the organic acid content combined with malic/citric were used for PCA, suggesting that malic/citric might be an important index for differentiation and authentication of juices of apple, pear, peach, grape, strawberry, blueberry, and sweet cherry.

In addition, the combination of sugar and organic acid composition was used as analytical data for PCA. Results showed that all the seven groups of fruit juice samples were separated distinctly in the score plots, indicating a satisfactory differentiation of these fruit juices according to species (Figure 1(e)). As compared with other datasets, the combination of sugar and organic acid composition resulted in a more distinct recognizing pattern for the differentiation of these seven species of fruit juice. 

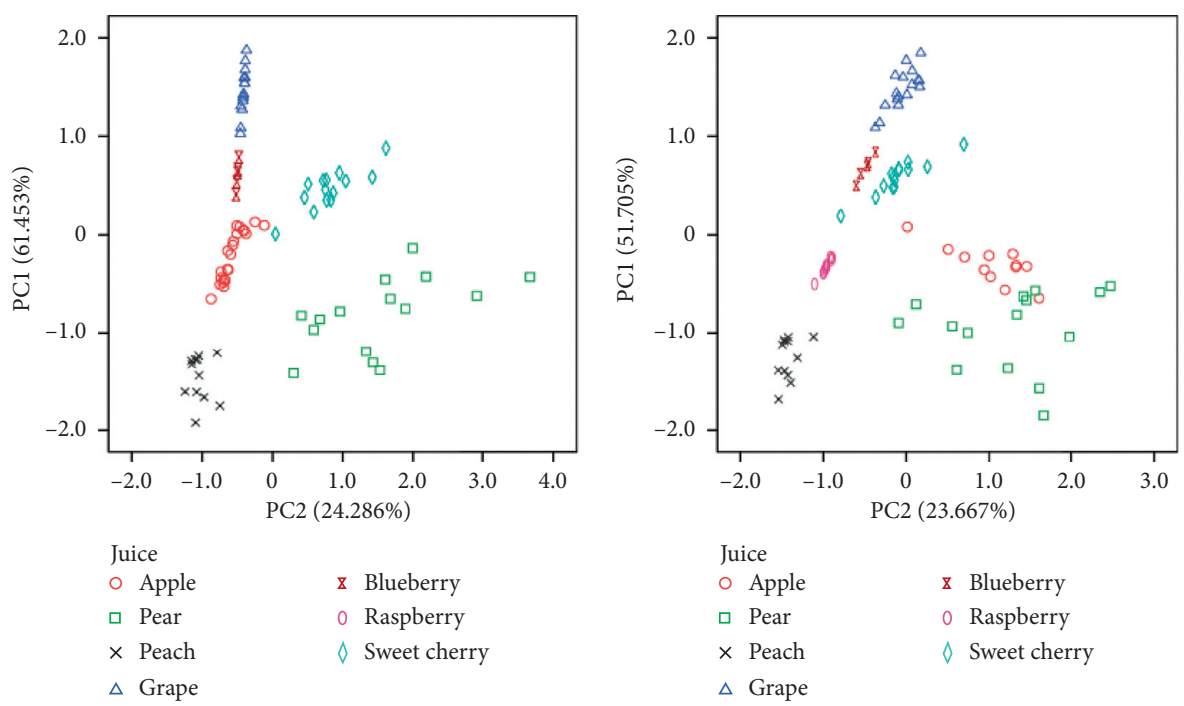

(a)

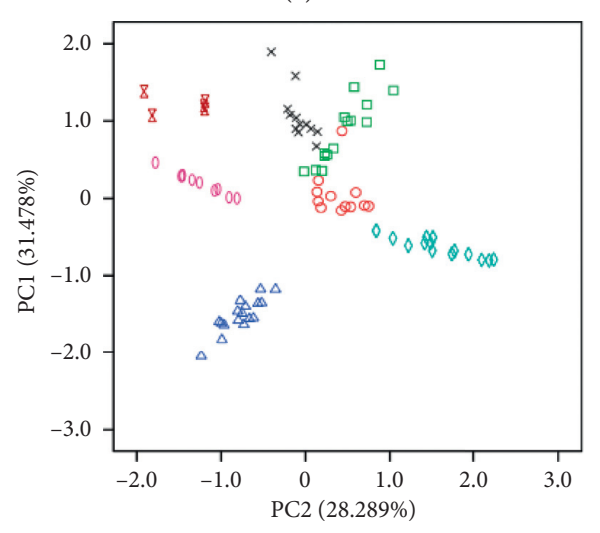

(b)

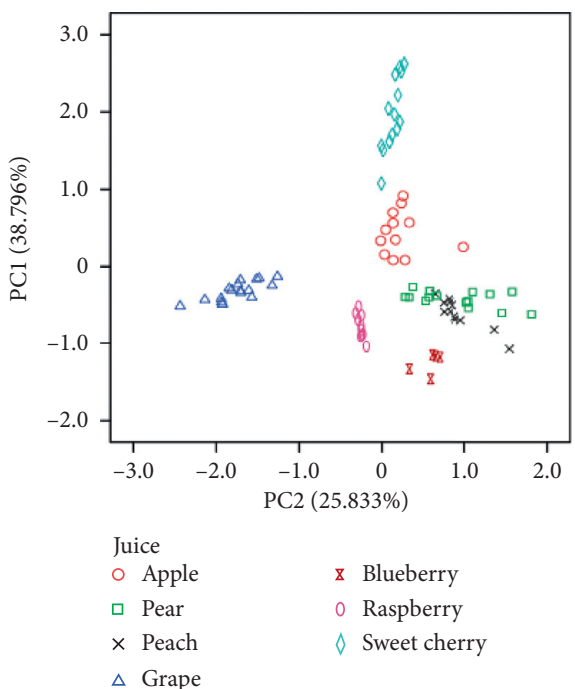

(c)

(d)

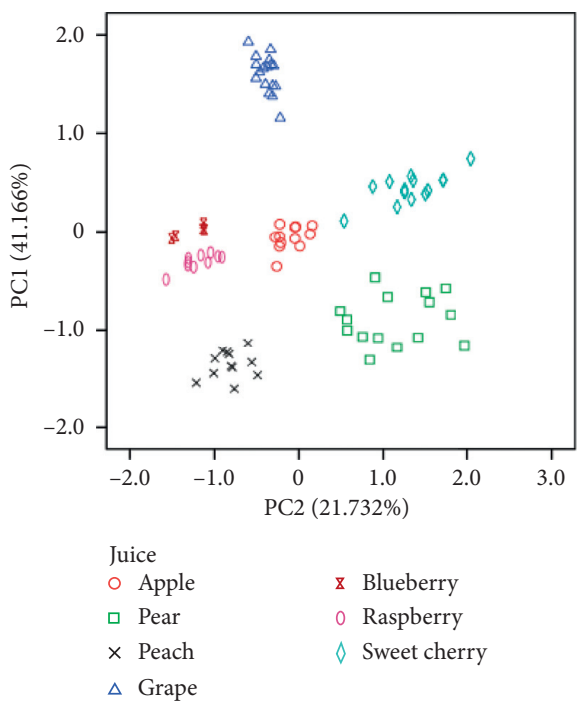

(e)

FIGURE 1: Score plots of PC1 vs. PC2 obtained with all analyzed samples used as analytical data for PCA: (a) sugar content; (b) sugar content combined with Fru/Glu; (c) organic acid content; (d) organic acid content combined with malic/citric; (e) sugar content combined with organic acid content. 


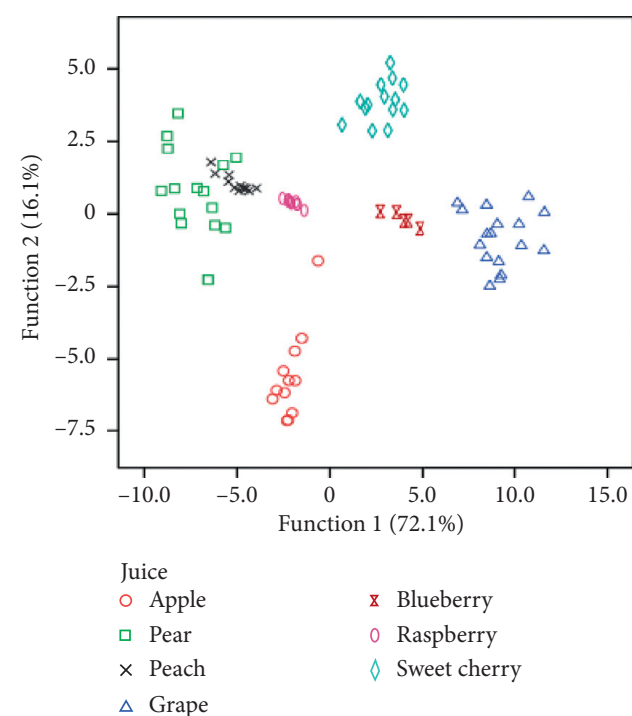

(a)

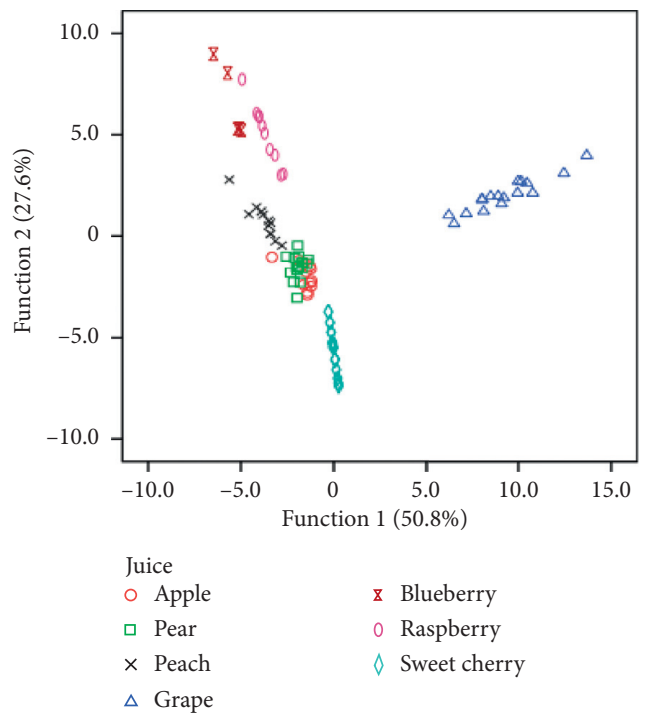

(c)

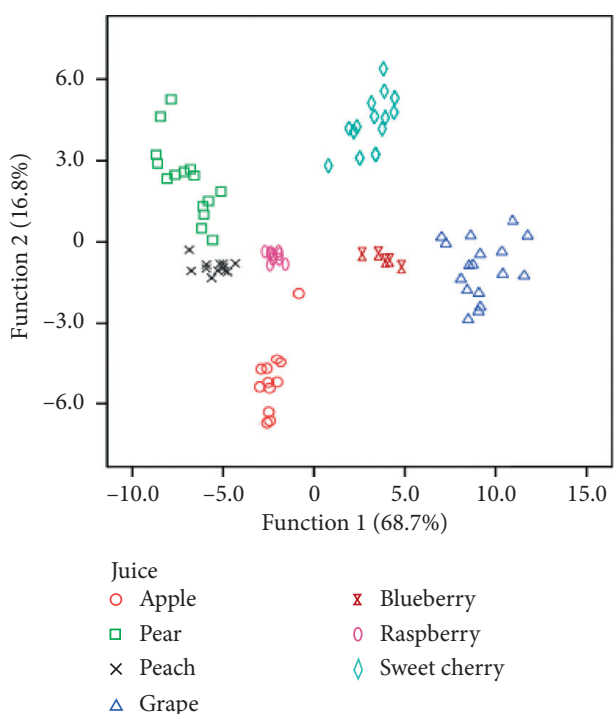

(b)

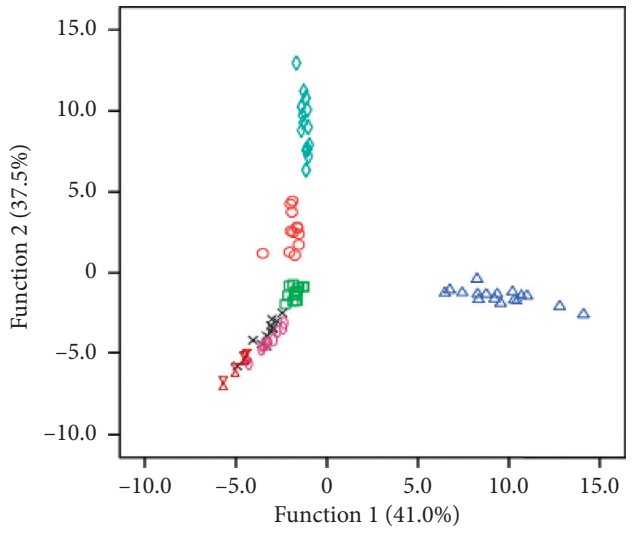

Juice
- Apple
z Blueberry
$\square$ Pear
0 Raspberry
$\times$ Peach
$\triangle$ Sweet cherry

(d)

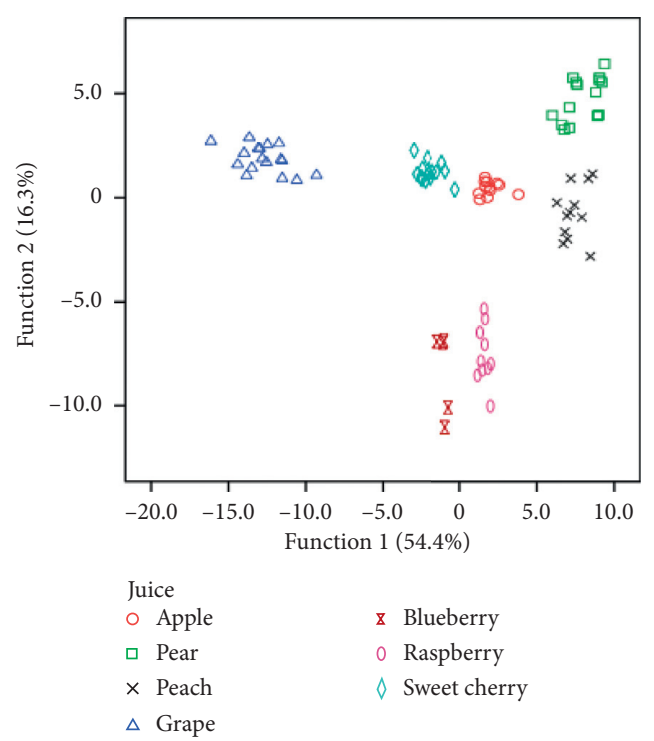

(e)

Figure 2: Score plots of Function 1 vs. Function 2 obtained with all analyzed samples used as analytical data for LDA: (a) sugar content; (b) sugar content combined with Fru/Glu; (c) organic acid content; (d) organic acid content combined with malic/citric; (e) sugar content combined with organic acid content. 
TABLE 3: Correct classification rate by LDA with different datasets of predictors.

\begin{tabular}{lcc}
\hline Dataset & Original (\%) & Cross validation (\%) \\
\hline Sugar content & 98.8 & 98.8 \\
Sugar content + Fru/Glu & 98.8 & 98.8 \\
Organic acid content & 95.3 & 94.1 \\
Organic acid content + malic/citric & 100 & 98.8 \\
Sugar and organic acid content & 100 & 100 \\
\hline
\end{tabular}

TABLE 4: Discrimination effect of adulterate samples by LDA with sugar and organic acid content.

\begin{tabular}{|c|c|c|}
\hline Fruit juice & Adulterate & Discrimination effect \\
\hline \multirow{3}{*}{ Sweet cherry } & With $20 \%, 40 \%, 60 \%$, and $80 \%$ of apple juice & All \\
\hline & With $20 \%, 40 \%, 60 \%$, and $80 \%$ of pear juice & All \\
\hline & With $20 \%, 40 \%, 60 \%$, and $80 \%$ of peach juice & All \\
\hline \multirow{3}{*}{ Blueberry } & With $20 \%, 40 \%, 60 \%$, and $80 \%$ of apple juice & All \\
\hline & With $20 \%, 40 \%, 60 \%$, and $80 \%$ of pear juice & All \\
\hline & With $20 \%, 40 \%, 60 \%$, and $80 \%$ of peach juice & All \\
\hline \multirow{3}{*}{ Raspberry } & With $20 \%, 40 \%, 60 \%$, and $80 \%$ of apple juice & All \\
\hline & With $20 \%, 40 \%, 60 \%$, and $80 \%$ of pear juice & All \\
\hline & With $20 \%, 40 \%, 60 \%$, and $80 \%$ of peach juice & All \\
\hline \multirow{3}{*}{ Grape } & With $20 \%, 40 \%, 60 \%$, and $80 \%$ of apple juice & Except for adulterate with $20 \%$ of apple juice \\
\hline & With $20 \%, 40 \%, 60 \%$, and $80 \%$ of pear juice & Except for adulterate with $20 \%$ of pear juice \\
\hline & With $20 \%, 40 \%, 60 \%$, and $80 \%$ of peach juice & All \\
\hline
\end{tabular}

3.4. Supervised Classification of Fruit Juices by Linear Discriminant Analysis. With the aim to classify the fruit juice samples according to the species they belong to, the most popular supervised classification method, linear discriminant analysis (LDA), was performed based on the analytical data of sugar and/or organic acid composition obtained from all the 85 juice samples.

As shown in Figure 2 and Table 3, the combination of sugar and organic acid composition gave a satisfactory classification of the 85 fruit juice samples according to species, with a $100 \%$ correct classification rate for both the original and the cross-validation method. The worst classification was obtained when the data of the organic acid content were used for LDA, with a correct classification rate of $95.3 \%$ for the original and $94.1 \%$ for the cross-validation method. However, the correct classification rate was improved to $100 \%$ for the original and $98.8 \%$ for the crossvalidation method, respectively, when the data of malic/ citric were added into the dataset of predictors, in which only one apple juice sample was misclassified in the peach juice group. These results are consistent with those of PCA, which confirms the important role of malic/citric for differentiation and authentication of juices of these seven species of fruit juice. As the data of sugar composition were subjected to LDA, the correct classification rate for the original and the cross-validation method achieved $98.8 \%$, in which only one apple juice sample was misclassified in the strawberry juice group. These results indicate that the combination of sugar and organic acid composition is superior to other datasets which comprise sugar or organic acid composition alone. The combination of different datasets (two or three of conventional quality parameters, volatile compounds, and minerals) for LDA was also performed in differentiation of cherries according to the cultivar, and a more excellent separation of cultivars was achieved as compared with those using an individual dataset of conventional quality parameters, volatile compounds, or minerals [40].

3.5. Discrimination of Adulteration of Higher-Cost Fruit Juices with Cheaper Alternatives by LDA. In order to assess the possibility of detecting adulteration of higher-cost fruit juices with cheaper alternatives, the soluble sugar and organic acid composition in the fruit juice blends were analyzed and subjected to LDA in combination with the data of each corresponding pure juices, and the discrimination effect of adulterate samples from each corresponding pure juice is summarized in Table 4. Except for grape juice adulterates with 20\% of apple juice and pear juice, all other adulteration of sweet cherry juice, blueberry juice, raspberry juice, and grape juice with the addition of apple juice, pear juice, or peach juice could be distinguished from their corresponding pure juices. These preliminary results confirm the idea that the sugar and organic acid profiles may be a useful approach for detecting adulteration of higher-cost fruit juices with cheaper alternatives. NavarroPascual-Ahuir et al. $[5,6]$ also noted the possibility of detecting the percentages of orange and pineapple juices in binary blends with grape juice by using sugar or organic acid composition. However, the juice samples of the present research were still limited, and more other commercial fruit juices from different seasons and areas should be included in the further research.

\section{Conclusions}

In spite of the great differences in contents of individual sugar and organic acid among different varieties, the distribution of each sugar and organic acid in juices showed 
some specific characteristics related to fruit species, which can afford the differentiation of apple, pear, peach, grape, sweet cherry, strawberry, and blueberry juice. Based on the sucrose content and their proportion in the total sugar content, the seven species of fruit juice could be classified to three categories: one is peach juice, which represents a high content of sucrose in total sugar; another class includes apple, pear, and strawberry juice, with a medium content of sucrose in total sugar; the other three fruit juices (grape, sweet cherry, and blueberry) were characterized as few sucrose content in soluble sugars. With the aid of principal component analysis and linear discriminant analysis, the juice samples can be successfully classified according to fruit species by using the sugar and/or organic acid composition as analytical data. Among different datasets for PCA and LDA, the combination of sugar and organic acid composition gives the best differentiation of these seven species of fruit juice, with a $100 \%$ correct classification rate for both the original and the cross-validation method in LDA. Sugar profile is superior to the organic acid profile for the differentiation of these seven species of fruit juice. In addition, the combination of the organic acid content and malic/citric may also improve the differentiation of these seven species of fruit juice as compared with the individual dataset of the organic acid content. Furthermore, the sugar and organic acid profiles may be a useful approach for detecting adulteration of higher-cost fruit juices with cheaper alternatives.

\section{Data Availability}

The data used to support the findings of this study are available from the corresponding author upon request.

\section{Conflicts of Interest}

The authors declare that there are no conflicts of interest.

\section{Acknowledgments}

This work was funded by the Science and Technology Research Project of Henan Province (182102110035) and the Agricultural Science and Technology Innovation Program (ASTIP) of the Chinese Academy of Agricultural Sciences (CAAS-ASTIP-2016-ZFRI).

\section{Supplementary Materials}

Table S1: sugar and organic acid content of apple juice of different varieties; Table S2: sugar and organic acid content of pear juice of different varieties; Table S3: sugar and organic acid content of peach juice of different varieties; Table S4: sugar and organic acid content of grape juice of different varieties; Table S5: sugar and organic acid content of sweet cherry juice of different varieties; Table S6: sugar and organic acid content of strawberry juice of different varieties; Table S7: sugar and organic acid content of blueberry juice of different varieties; Figure S1: HPLC chromatogram of sugar and organic acid standards. (Supplementary Materials)

\section{References}

[1] K. L. Bett-Garber, J. M. Lea, M. A. Watson et al., "Flavor of fresh blueberry juice and the comparison to amount of sugars, acids, anthocyanidins, and physicochemical measurements," Journal of Food Science, vol. 80, no. 4, pp. S818-S827, 2015.

[2] J. Yi, B. T. Kebede, D. N. Hai Dang et al., "Quality change during high pressure processing and thermal processing of cloudy apple juice,” Lwt, vol. 75, pp. 85-92, 2017.

[3] R. Fügel, R. Carle, and A. Schieber, "Quality and authenticity control of fruit purées, fruit preparations and jams-a review," Trends in Food Science \& Technology, vol. 16, no. 10, pp. 433-441, 2005.

[4] K. Marszalek, L. Wozniak, F. J. Barba et al., "Enzymatic, physicochemical, nutritional and phytochemical profile changes of apple (Golden Delicious L.) juice under supercritical carbon dioxide and long-term cold storage," Food Chemistry, vol. 268, pp. 279-286, 2018.

[5] M. Navarro-Pascual-Ahuir, M. J. Lerma-García, E. F. SimóAlfonso, and J. M. Herrero-Martínez, "Rapid differentiation of commercial juices and blends by using sugar profiles obtained by capillary zone electrophoresis with indirect UV detection," Journal of Agricultural and Food Chemistry, vol. 63, no. 10, pp. 2639-2646, 2015.

[6] M. Navarro-Pascual-Ahuir, M. J. Lerma-García, E. F. SimóAlfonso, and J. M. Herrero-Martínez, "Quality control of fruit juices by using organic acids determined by capillary zone electrophoresis with poly(vinyl alcohol)-coated bubble cell capillaries," Food Chemistry, vol. 188, pp. 596-603, 2015.

[7] M. Navarro-Pascual-Ahuir, M. J. Lerma-García, E. F. SimóAlfonso, and J. M. Herrero-Martínez, "Analysis of aliphatic organic acids in commercial fruit juices by capillary electrophoresis with indirect UV detection: application to differentiation of fruit juices," Food Analytical Methods, vol. 10, no. 12, pp. 3991-4002, 2017.

[8] C. Nikolaou, I. K. Karabagias, I. Gatzias, S. Kontakos, A. Badeka, and M. G. Kontominas, "Differentiation of fresh greek orange juice of the merlin cultivar according to geographical origin based on the combination of organic acid and sugar content as well as physicochemical parameters using chemometrics," Food Analytical Methods, vol. 10, no. 7, pp. 2217-2228, 2017.

[9] A. M. Zakharova, I. L. Grinshtein, and L. A. Kartsova, "Determination of carbohydrates and sweeteners in foods and biologically active additives by high-performance liquid chromatography," Journal of Analytical Chemistry, vol. 68, no. 12, pp. 1081-1084, 2013.

[10] V. Ivanova-Petropulos, D. Petruševa, and S. Mitrev, "Rapid and simple method for determination of target organic acids in wine using HPLC-DAD analysis," Food Analytical Methods, vol. 13, no. 5, pp. 1078-1087, 2020.

[11] P. Mattila and J. Kumpulainen, "Determination of free and total phenolic acids in plant-derived foods by HPLC with diode-array detection," Journal of Agricultural and Food Chemistry, vol. 50, no. 13, pp. 3660-3667, 2002.

[12] S. Ulusoy and M. Akçay, "Simultaneous determination of vitamins B1 and B2 in food samples by modified cloud point extraction method and HPLC-DAD," Food Analytical Methods, vol. 11, no. 1, pp. 260-269, 2018.

[13] A. A. F. Zielinski, C. M. Braga, I. M. Demiate, F. L. Beltrame, A. Nogueira, and G. Wosiacki, "Development and optimization of a HPLC-RI method for the determination of major sugars in apple juice and evaluation of the effect of the 
ripening stage," Food Science and Technology, vol. 34, no. 1, pp. 38-43, 2014.

[14] E. M. Coelho, C. V. Da Silva Padilha, G. A. Miskinis et al., "Simultaneous analysis of sugars and organic acids in wine and grape juices by HPLC: method validation and characterization of products from northeast Brazil," Journal of Food Composition and Analysis, vol. 66, pp. 160-167, 2018.

[15] P. Legua, P. Melgarejo, J. J. Martínez, R. Martínez, and F. Hernández, "Evaluation of Spanish pomegranate juices: organic acids, sugars, and anthocyanins," International Journal of Food Properties, vol. 15, no. 3, pp. 481-494, 2012.

[16] H. Kelebek, S. Selli, A. Canbas, and T. Cabaroglu, "HPLC determination of organic acids, sugars, phenolic compositions and antioxidant capacity of orange juice and orange wine made from a Turkish cv. Kozan," Microchemical Journal, vol. 91, no. 2, pp. 187-192, 2009.

[17] I. Nishiyama, T. Fukuda, A. Shimohashi, and T. Oota, "Sugar and organic acid composition in the fruit juice of different actinidia varieties," Food Science and Technology Research, vol. 14, no. 1, pp. 67-73, 2008.

[18] F. Karadeniz and A. Ekşi, "Sugar composition of apple juices," European Food Research and Technology, vol. 215, no. 2, pp. 145-148, 2002.

[19] N. Hasnaoui, R. Jbir, M. Mars et al., "Organic acids, sugars, and anthocyanins contents in juices of Tunisian pomegranate fruits," International Journal of Food Properties, vol. 14, no. 4, pp. 741-757, 2011.

[20] M. Turkyilmaz, "“'Anthocyanin and organic acid profiles of pomegranate (Punica granatum L.) juices from registered varieties in Turkey," International Journal of Food Science and Technology, vol. 48, no. 10, pp. 2086-2095, 2013.

[21] F. Saidani, R. Giménez, C. Aubert, G. Chalot, J. A. Betrán, and Y. Gogorcena, "Phenolic, sugar and acid profiles and the antioxidant composition in the peel and pulp of peach fruits," Journal of Food Composition and Analysis, vol. 62, pp. 126133, 2017.

[22] P. Nowicka, A. Wojdyło, and P. Laskowski, "Principal component analysis (PCA) of physicochemical compounds' content in different cultivars of peach fruits, including qualification and quantification of sugars and organic acids by HPLC," European Food Research and Technology, vol. 245, no. 4, pp. 929-938, 2019.

[23] O. T. Okan, İ. Deniz, N. Yayli, İ. G. Şat, M. Öz, and G. Hatipoğlu Serdar, "Antioxidant activity, sugar content and phenolic profiling of blueberries cultivars: a comprehensive comparison," Notulae Botanicae Horti Agrobotanici ClujNapoca, vol. 46, no. 2, pp. 639-652, 2018.

[24] H.-F. Liu, B.-H. Wu, P.-G. Fan, S.-H. Li, and L.-S. Li, "Sugar and acid concentrations in 98 grape cultivars analyzed by principal component analysis," Journal of the Science of Food and Agriculture, vol. 86, no. 10, pp. 1526-1536, 2006.

[25] A. A. Hayaloglu and N. Demir, "'Physicochemical characteristics, antioxidant activity, organic acid and sugar contents of 12 sweet cherry (Prunus avium L.) cultivars grown in Turkey," Journal of Food Science, vol. 80, no. 3, pp. C564-C570, 2015.

[26] E. Muntean, "Simultaneous carbohydrate chromatography and unsuppressed ion chromatography in detecting fruit juices adulteration," Chromatographia, vol. 71, no. S1, pp. S69-S74, 2010.

[27] A. Eksi and S. Karav, "Sorbitol/xylitol level in fruit juices obtained from different fruit species and its importance in terms of authenticity control," Fruit Processing, vol. 21, no. 3, pp. 94-97, 2011.
[28] F. R. Spinelli, S. V. Dutra, G. Carnieli, S. Leonardelli, A. P. Drehmer, and R. Vanderlinde, "Detection of addition of apple juice in purple grape juice," Food Control, vol. 69, pp. 1-4, 2016.

[29] P. Thavarajah and N. H. Low, "Adulteration of apple with pear juice: emphasis on major carbohydrates, proline, and arbutin," Journal of Agricultural and Food Chemistry, vol. 54, no. 13, pp. 4861-4867, 2006.

[30] Y. Soyer, N. Koca, and F. Karadeniz, "Organic acid profile of Turkish white grapes and grape juices," Journal of Food Composition and Analysis, vol. 16, no. 5, pp. 629-636, 2003.

[31] M. D. S. Lima, I. D. S. V. Silani, I. M. Toaldo et al., "Phenolic compounds, organic acids and antioxidant activity of grape juices produced from new Brazilian varieties planted in the Northeast Region of Brazil," Food Chemistry, vol. 161, pp. 94-103, 2014.

[32] S. Ehling and S. Cole, "Analysis of organic acids in fruit juices by liquid Chromatography-Mass Spectrometry: an enhanced tool for authenticity testing," Journal of Agricultural and Food Chemistry, vol. 59, no. 6, pp. 2229-2234, 2011.

[33] H. Y. Gao, S. G. Wang, and X. S. Hu, "“Study on determination of kinds and contents of organic acids in pear juice by high performance liquid chromatography," Food and Fermentation Industries, vol. 30, no. 8, pp. 96-100, 2004.

[34] F. A. Ayaz, A. Kadioglu, E. Bertoft, C. Acar, and I. Turna, "Effect of fruit maturation on sugar and organic acid composition in two blueberries (Vaccinium arctostaphylos and V. myrtillus) native to Turkey," New Zealand Journal of Crop and Horticultural Science, vol. 29, no. 2, pp. 137-141, 2001.

[35] H. Bae, S. Yun, J. Jun et al., "Assessment of organic acid and sugar composition in apricot, plumcot, plum, and peach during fruit development," Journal of Applied Botany and Food Quality, vol. 87, pp. 24-29, 2014.

[36] A. Begic-Akagic, N. Spaho, F. Gasi et al., "Sugar and organic acid profiles of the traditional and international apple cultivars for processing," Journal of Hygienic Engineering and Design, vol. 7, pp. 190-196, 2014.

[37] V. Usenik, J. Fabčič, and F. Š.bnikd, "Sugars, organic acids, phenolic composition and antioxidant activity of sweet cherry (Prunus avium L.)," Food Chemistry, vol. 107, no. 1, pp. 185-192, 2008.

[38] V. Gökmen and J. Acar, "Fumaric acid in apple juice: a potential indicator of microbial spoilage of apples used as raw material," Food Additives and Contaminants, vol. 21, no. 7, pp. 622-631, 2004.

[39] M. Esteki, J. Simal-Gandara, Z. Shahsavari, S. Zandbaaf, E. Dashtaki, and Y. Vander Heyden, "A review on the application of chromatographic methods, coupled to chemometrics, for food authentication," Food Control, vol. 93, pp. 165-182, 2018.

[40] S. Papapetros, A. Louppis, I. Kosma, S. Kontakos, A. Badeka, and M. G. Kontominas, "Characterization and differentiation of botanical and geographical origin of selected popular sweet cherry cultivars grown in Greece," Journal of Food Composition and Analysis, vol. 72, pp. 48-56, 2018. 